\title{
COMPOSITION OPERATORS IN ORLICZ SPACES
}

\author{
YUNAN CUI, HENRYK HUDZIK, ROMESH KUMAR and LECH MALIGRANDA
}

(Received 25 June 2002; revised 5 February 2003)

Communicated by A. Pryde

\begin{abstract}
Composition operators $C_{\tau}$ between Orlicz spaces $L^{\varphi}(\Omega, \Sigma, \mu)$ generated by measurable and nonsingular transformations $\tau$ from $\Omega$ into itself are considered. We characterize boundedness and compactness of the composition operator between Orlicz spaces in terms of properties of the mapping $\tau$, the function $\varphi$ and the measure space $(\Omega, \Sigma, \mu)$. These results generalize earlier results known for $L^{p}$-spaces.
\end{abstract}

2000 Mathematics subject classification: primary 47B33, 46E30; secondary 47B07, 46B70.

Keywords and phrases: composition operators, Orlicz spaces, compact operators, interpolation.

\section{Introduction}

Let $\Omega=(\Omega, \Sigma, \mu)$ be a $\sigma$-finite complete measure space and let $\tau: \Omega \rightarrow \Omega$ be a measurable transformation, that is, $\tau^{-1}(A) \in \Sigma$ for any $A \in \Sigma$. If $\mu\left(\tau^{-1}(A)\right)=0$ for all $A \in \Sigma$ with $\mu(A)=0$, then $\tau$ is said to be nonsingular. This condition means that the measure $\mu \circ \tau^{-1}$, defined by $\mu \circ \tau^{-1}(A):=\mu\left(\tau^{-1}(A)\right)$ for $A \in \Sigma$, is absolutely continuous with respect to $\mu$ (it is usually denoted $\mu \circ \tau^{-1} \ll \mu$ ). Then the Radon-Nikodým theorem ensures the existence of a non-negative locally integrable function $f_{\tau}$ on $\Omega$ such that $\mu \circ \tau^{-1}(A)=\int_{A} f_{\tau}(t) d \mu(t)$ for $A \in \Sigma$.

Any measurable nonsingular transformation $\tau$ induces a linear operator (composition operator) $C_{\tau}$ from $L^{0}(\Omega)$ into itself defined by

$$
C_{\tau} x(t)=x(\tau(t)), \quad t \in \Omega, x \in L^{0}(\Omega),
$$

where $L^{0}(\Omega)$ denotes the linear space of all equivalence classes of $\Sigma$-measurable functions on $\Omega$, where we identify any two functions that are equal $\mu$-almost everywhere on $\Omega$.

(C) 2004 Australian Mathematical Society $1446-7887 / 04 \$ A 2.00+0.00$ 
Here the nonsingularity of $\tau$ guarantees that the operator $C_{\tau}$ is well defined as a mapping of equivalence classes of functions into itself since $x=y \mu$-a.e. implies $C_{\tau} x=C_{\tau} y \mu$-a.e.

If $C_{\tau}$ maps an Orlicz space $L^{\varphi}(\Omega)$ into itself, then we call $C_{\tau}$ a composition operator in $L^{\varphi}(\Omega)$. Note that, in this case, $C_{\tau}$ as a positive linear operator in $L^{\varphi}(\Omega)$ is bounded.

Boundedness of composition operators in $L^{p}(\Omega)$-spaces $(1 \leq p<\infty)$ for finite measures appeared already in the Dunford-Schwartz book [4, Lemma 7, pages 664665] and for $\sigma$-finite measures in Singh's paper [21] (the sequence case in [23]) and also in the book [25]. Namely, a measurable transformation $\tau$ induces a bounded composition operator $C_{\mathrm{r}}$ in $L^{p}(\Omega)$ for $1 \leq p<\infty$ if and only if $\mu \circ \tau^{-1} \ll \mu$ and the Radon-Nikodým derivative $d \mu \circ \tau^{-1} / d \mu=f_{\tau}$ is essentially bounded on $\Omega$. The last two conditions can be written as one: there exists a constant $K>0$ such that

$$
\mu\left(\tau^{-1}(A)\right) \leq K \mu(A) \text { for all } A \in \Sigma
$$

Moreover,

$$
\left\|C_{\tau}\right\|_{L^{p} \rightarrow L^{p}}=\left(\sup _{A \in \Sigma, 0<\mu(A)<\infty} \mu\left(\tau^{-1}(A)\right) / \mu(A)\right)^{1 / p} .
$$

Observe that the composition operator $C_{\tau}$ is a bounded operator in $L^{\infty}(\mu)$ with norm 1 , that is, a nonexpansive mapping for any measurable nonsingular transformation $\tau$.

In the case that $\varphi$ is an $N$-function, some results on boundedness of composition operators in the Orlicz space $L^{\varphi}(\Omega)$ were obtained in [9, Theorem 2.1] (see also [16]).

Compactness results of composition operator were proved by Singh [20] and SinghKumar [24, 22] in $L^{2}(\Omega)$-space. Then Petrović [15], Xu [28] and Takagi [26] extended them to the $L^{p}(\Omega)$-spaces with $1 \leq p \leq \infty$. It turns out that there is no compact composition operator on $L^{p}(\Omega)$, when $\mu$ is either non-atomic or purely atomic with all atoms of equal measure. But there are some weighted sequence $l^{p}$-spaces which do have compact composition operators.

We will present some new results on boundedness and compactness of composition operators in Orlicz spaces.

We need some notions from Orlicz spaces. Let $\varphi:[0, \infty) \rightarrow[0, \infty]$ be an Orlicz function, that is, a convex function such that $\varphi(0)=0, \varphi(u) \rightarrow \infty$ as $u \rightarrow \infty$ and which is not identically zero or infinity on $(0, \infty)$. Assume also that $\varphi$ is leftcontinuous at $b_{\varphi}$, where $b_{\varphi}=\sup \{u>0: \varphi(u)<\infty\}$. Note that if $b_{\varphi}<\infty$, then we can have two cases $\varphi\left(b_{\varphi}\right)<\infty$ or $\varphi\left(b_{\varphi}\right)=\infty$. Another important constant connected with the Orlicz function $\varphi$ is $a_{\varphi}=\inf \{u>0: \varphi(u)>0\}$. Of course, $0 \leq a_{\varphi} \leq b_{\varphi} \leq \infty$. For $x \in L^{0}(\Omega)$, define the modular

$$
I_{\varphi}(x)=\int_{\Omega} \varphi(|x(s)|) d \mu(s)
$$


and the Orlicz space

$$
L^{\varphi}(\Omega)=L^{\varphi}(\Omega, \Sigma, \mu)=\left\{x \in L^{0}(\Omega): I_{\varphi}(\lambda x)<\infty \text { for some } \lambda=\lambda(x)>0\right\} .
$$

This space is a Banach space with two norms: the Luxemburg-Nakano norm

$$
\|x\|_{\varphi}=\inf \left\{\lambda>0: I_{\varphi}(x / \lambda) \leq 1\right\}
$$

and the Orlicz norm (in the Amemiya form)

$$
\|x\|_{\varphi}^{0}=\inf _{k>0}\left(1+I_{\varphi}(k x)\right) / k .
$$

It is well known that $\|x\|_{\varphi} \leq\|x\|_{\varphi}^{0} \leq 2\|x\|_{\varphi}$ and $\|x\|_{\varphi} \leq 1$ if and only if $I_{\varphi}(x) \leq 1$ (see for example [7, page 80]). Moreover, if $A \in \Sigma$ and $0<\mu(A)<\infty$, then $\left\|\chi_{A}\right\|_{\varphi}=1 / \varphi^{-1}(1 / \mu(A))$, where $\varphi^{-1}(t)=\inf \{s>0: \varphi(s)>t\}$ is the rightcontinuous inverse of $\varphi$. Note that the equality of the Orlicz norm and the Amemiya norm was recently proved in [5]. Before only an estimate of the Orlicz norm from above by the Amemiya norm was known (see [13, Theorem 1.2.6]) with equality when both $\varphi$ and its complementary function $\varphi^{*}$ are $\mathrm{N}$-functions (see [7, Theorem III.10.4], [11, Theorem 8.6] and [17, Theorem III.3.13]) .

The Orlicz space $L^{\varphi}(\Omega)$ with each of the above two norms is a rearrangementinvariant space, that is, a symmetric space with the Fatou property (see [1, Theorem 8.9], [8, page 104]).

We say that an Orlicz function $\varphi$ satisfies the condition $\Delta_{2}$ for all $u$ (respectively, for large $u$; for small $u$ ) if there exists a positive constant $K$ (respectively, a positive $K$ and $u_{0}>0$ with $\varphi\left(u_{0}\right)<\infty$; a positive constant $K$ and $u_{0}>0$ with $\varphi\left(u_{0}\right)>0$ ) such that $\varphi(2 u) \leq K \varphi(u)$ for all $u>0$ (respectively, for all $u \geq u_{0}$; for all $0<u \leq u_{0}$ ).

If $\varphi$ satisfies the condition $\Delta_{2}$ for all $u$, then for any $\sigma$-finite measure space the dual of the Orlicz space $L^{\varphi}(\Omega)$ is the Orlicz space $L^{\varphi^{*}}(\Omega)$ generated by the complementary function $\varphi^{*}$ of $\varphi$, defined by $\varphi^{*}(u):=\sup _{v>0}(u v-\varphi(v))$. Moreover,

$$
\left(L^{\varphi}(\Omega),\|\cdot\|_{\varphi}\right)^{*}=\left(L^{\varphi^{*}}(\Omega),\|\cdot\|_{\varphi^{*}}^{0}\right) \quad \text { and } \quad\left(L^{\varphi}(\Omega),\|\cdot\|_{\varphi}^{0}\right)^{*}=\left(L^{\varphi^{*}}(\Omega),\|\cdot\|_{\varphi^{*}}\right) .
$$

The paper is organized as follows. In Section 2 we study modular continuity and norm continuity of composition operators $C_{\mathrm{r}}$ in Orlicz spaces $L^{\varphi}(\Omega)$. We were able to completely characterize modular continuity of $C_{\tau}$. It is surprising that the necessary and sufficient conditions for modular continuity do not depend on Orlicz functions $\varphi$ but only on the transformation $\tau$. Our results on norm continuity of $C_{\tau}$ are also complete. The form of the necessary and sufficient conditions for the norm continuity established in this paper depends on the regularity of the Orlicz function $\varphi$. In the case when $\varphi$ satisfies the condition $\Delta_{2}$ for all $u>0$, the conditions have the same form as for the modular continuity, so they do not depend on $\varphi$, for any measure $\mu$. If $\mu$ is 
nonatomic and finite the same is true if $\varphi$ satisfies the condition $\Delta_{2}$ for large values of $u>0$. If the measure $\mu$ is nonatomic and infinite, then the condition $\Delta_{2}$ for large values of $u>0$ is enough to explain the necessary and sufficient condition for the continuity of $C_{\tau}$ from $L^{\varphi}(\Omega)$ into itself in terms of $\varphi$. Without any regularity condition for $\varphi$, and without any restriction on the measure $\mu$ it is established that the composition operator $C_{\tau}$ generated by a nonsingular transformation $\tau$ is continuous from an Orlicz spaces $L^{\varphi}(\Omega)$ into itself if and only if $L^{\varphi}(\Omega)$ is contained in the Musielak-Orlicz space $L^{\psi}(\Omega)$, where $\psi(t, u)=\varphi(u) w(t)$ and $w$ is the Radon-Nikodým derivative $d \mu \circ \tau^{-1} / d \mu$. It means that in the general case we were not able to explain the conditions directly in terms of the generating function $\varphi$.

In Section 3, we investigate compactness of composition operators in Orlicz spaces. As in the $L^{p}$-case, compactness of a composition operator $C_{\tau}$ in the Orlicz space $L^{\varphi}(\Omega)$ implies that the underlying measure $\mu$ is purely atomic. Under some assumptions on the function $\varphi$ and the atoms of $\mu$, we prove necessary and sufficient conditions for $C_{\tau}$ to be compact. In particular, we can have compactness of the composition operator in weighted Orlicz sequence spaces.

\section{Modular and norm continuity of composition operators}

For the modular continuity of the composition operator $C_{\tau}$ in an Orlicz space $L^{\varphi}(\Omega)$, we present necessary and sufficient conditions for any Orlicz function $\varphi$ and any $\sigma$-finite measure space $(\Omega, \Sigma, \mu)$. For any Orlicz function $\varphi$ which satisfies the condition $\Delta_{2}$ for all $u$, the same is done for norm continuity of the composition operator $C_{\tau}$ in $L^{\varphi}(\Omega)$. If $\varphi$ satisfies the condition $\Delta_{2}$ for large $u$, then the problem of continuity of the composition operator $C_{\tau}$ in $L^{\varphi}(\Omega)$ is completely solved if the measure space is nonatomic of finite or infinite measure. Without any regularity condition on $\varphi$, the conditions for continuity of $C_{\tau}$ from $L^{\varphi}(\Omega)$ into itself are explained in terms of the Radon-Nikodým derivative $d \mu \circ \tau^{-1} / d \mu$.

THEOREM 2.1. Assume that $\tau: \Omega \rightarrow \Omega$ is a measurable nonsingular transformation.

(a) If $0<a_{\varphi}=b_{\varphi}<\infty$, then $I_{\varphi}\left(C_{\tau} x\right)=I_{\varphi}(x)$ whenever $I_{\varphi}(x)<\infty$.

(b) If $0 \leq a_{\varphi}<b_{\varphi} \leq \infty$, then the inequality

$$
I_{\varphi}\left(C_{\tau} x\right) \leq K I_{\varphi}(x)
$$

holds for all $x$ such that $I_{\varphi}(x)<\infty$ with some $K>0$ independent of $x$ if and only if

$$
\mu\left(\tau^{-1}(A)\right) \leq K \mu(A)
$$

for all $A \in \Sigma$ with $\mu(A)<\infty$. 
PROOF. (a) In this case the function $\varphi$ is 0 on the interval $\left[0, a_{\varphi}\right)$ and $\infty$ on $\left(a_{\varphi}, \infty\right)$. Therefore, $I_{\varphi}(x)<\infty$ if and only if $\|x\|_{\infty} \leq a_{\varphi}$ and so $\left\|C_{\tau} x\right\|_{\infty} \leq a_{\varphi}$, which finally gives $I_{\varphi}\left(C_{\tau} x\right)=0=I_{\varphi}(x)$.

(b) Assume that $0 \leq a_{\varphi}<b_{\varphi} \leq \infty$.

Sufficiency. Assume that condition (2.2) is satisfied. It gives that $\mu \circ \tau^{-1} \ll \mu$ and by the Radon-Nikodým theorem, $\mu \circ \tau^{-1}(A)=\int_{A} f_{\tau}(t) d \mu(t)$ for $A \in \Sigma$ and for some function $f_{\tau}$ locally integrable on $\Omega$. Notice that $f_{\mathrm{r}} \in L^{\infty}(\Omega)$ and $\left\|f_{\tau}\right\|_{\infty} \leq K$. Otherwise, there is $A \in \Sigma$ with $0<\mu(A)<\infty$ such that $f_{\tau}(t)>K$ for any $t \in A$. This yields $\mu \circ \tau^{-1}(A)=\int_{A} f_{\tau}(t) d \mu(t)>K \mu(A)$, which contradicts condition (2.2).

Therefore,

$$
\begin{aligned}
I_{\varphi}\left(C_{\tau} x\right) & =\int_{\Omega} \varphi\left(\left|C_{\tau} x(s)\right|\right) d \mu(s)=\int_{\Omega} \varphi(|x(\tau(s))|) d \mu(s) \\
& =\int_{\tau(\Omega)} \varphi(|x(t)|) d\left(\mu \circ \tau^{-1}\right)(t) \leq \int_{\Omega} \varphi(|x(t)|) d\left(\mu \circ \tau^{-1}\right)(t) \\
& =\int_{\Omega} \varphi(|x(t)|) f_{\tau}(t) d \mu(t) \leq K \int_{\Omega} \varphi(|x(t)|) d \mu(t)=K I_{\varphi}(x) .
\end{aligned}
$$

Necessity. Assume that condition (2.1) holds. If $A \in \Sigma$ and $\mu(A)=0$, then the nonsingularity of $\tau$ yields $\mu\left(\tau^{-1}(A)\right)=0$ and we have equality in (2.2). Thus suppose that $A \in \Sigma$ and $0<\mu(A)<\infty$. Take $a \in\left(a_{\varphi}, b_{\varphi}\right)$ and set $x=a \chi_{A}$. Then

$$
I_{\varphi}(x)=\int_{A} \varphi(a) d \mu(s)=\varphi(a) \mu(A)<\infty .
$$

As $C_{\tau} \chi_{A}=\chi_{\tau^{-1}(A)}$, it follows by $(2.1)$ that

$$
\varphi(a) \mu\left(\tau^{-1}(A)\right)=I_{\varphi}\left(C_{\tau} x\right) \leq K I_{\varphi}(x)=K \varphi(a) \mu(A) .
$$

Since $0<\varphi(a)<\infty$, this gives $(2.2)$.

THEOREM 2.2. Assume that $\tau: \Omega \rightarrow \Omega$ is a measurable nonsingular transformation. Then the composition operator $C_{\tau}$ is bounded from an Orlicz space $L^{\varphi}(\Omega)$ into itself, that is, there exists $M>0$ such that

$$
\left\|C_{\tau} x\right\|_{\varphi} \leq M\|x\|_{\varphi} \text { for all } x \in L^{\varphi}(\Omega)
$$

if condition (2.2) holds. If, in addition, $\varphi$ satisfies the condition $\Delta_{2}$ for all $u>0$, then (2.3) and (2.2) are equivalent.

PROOF. Sufficiency. By Theorem 2.1 we know that if (2.2) is satisfied with $K \geq 1$, then (2.1) holds and

$$
I_{\varphi}\left(\frac{C_{\tau} x}{K\|x\|_{\varphi}}\right) \leq \frac{1}{K} I_{\varphi}\left(\frac{C_{\tau} x}{\|x\|_{\varphi}}\right) \leq I_{\varphi}\left(\frac{x}{\|x\|_{\varphi}}\right) \leq 1,
$$


whence $\left\|C_{\tau} x\right\|_{\varphi} \leq K\|x\|_{\varphi}$ for all $0 \neq x \in L^{\varphi}(\Omega)$, that is, (2.3) holds with $M=K$. Note that this part of the theorem holds without the $\Delta_{2}$-condition.

Necessity. Setting in inequality (2.3) $x=\chi_{A}$, where $A \in \Sigma$ and $0<\mu(A)<\infty$, we get the estimate

or equivalently

$$
\frac{1}{\varphi^{-1}\left(1 / \mu\left(\tau^{-1}(A)\right)\right)} \leq \frac{M}{\varphi^{-1}(1 / \mu(A))}
$$

$$
\varphi^{-1}\left(\frac{1}{\mu(A)}\right) \leq M \varphi^{-1}\left(\frac{1}{\mu\left(\tau^{-1}(A)\right)}\right)
$$

for all $A \in \Sigma$ with $0<\mu(A)<\infty$.

Since $\varphi$ satisfies the condition $\Delta_{2}$ for all $u>0$, it follows that

$$
L:=\sup _{u>0} \frac{\varphi(M u)}{\varphi(u)}<\infty,
$$

and $\varphi(M u) \leq L \varphi(u)$ for all $u>0$, which gives for $u=\varphi^{-1}(v)$ that

$$
\varphi\left(M \varphi^{-1}(v)\right) \leq L \varphi\left(\varphi^{-1}(v)\right) \leq L v
$$

and so

$$
M \varphi^{-1}(v) \leq \varphi^{-1}\left\{\varphi\left(M \varphi^{-1}(v)\right)\right\} \leq \varphi^{-1}(L v)
$$

for all $v>0$. Consequently, condition (2.4) yields

$$
\varphi^{-1}\left(\frac{1}{\mu(A)}\right) \leq M \varphi^{-1}\left(\frac{1}{\mu\left(\tau^{-1}(A)\right)}\right) \leq \varphi^{-1}\left(\frac{L}{\mu\left(\tau^{-1}(A)\right)}\right)
$$

or equivalently $\mu\left(\tau^{-1}(A)\right) \leq L \mu(A)$ for all $A \in \Sigma$ with $0<\mu(A)<\infty$, which finishes the proof of the necessity with $K=L$.

REMARK 1. Theorem 2.2 was, in fact, already formulated in [9] when $\varphi$ is an $\mathrm{N}$-function but in case of $M>1$ the proof was not complete.

REMARK 2. The sufficiency of Theorem 2.2 can be proved (again without the $\Delta_{2}$-condition) in two different ways, namely by using simple functions and by the Orlicz interpolation theorem which is saying that any Orlicz space $L^{\varphi}(\Omega)$ is an exact interpolation space between $L^{1}(\Omega)$ and $L^{\infty}(\Omega)$ (see [12]). We present below the second alternative proof.

ALTERNATIVE PROOF. Since $\tau$ is nonsingular, the operator $C_{\tau}$ is continuous between $L^{\infty}(\Omega)$ with $\left\|C_{\tau} x\right\|_{\infty} \leq\|x\|_{\infty}$ for all $x \in L^{\infty}(\Omega)$. Condition (2.2) yields that 
$C_{\tau}$ is continuous on $L^{1}(\Omega)$ with $\left\|C_{\tau} x\right\|_{1} \leq K\|x\|_{1}$ for all $x \in L^{1}(\Omega)$. By the Orlicz interpolation theorem (see [12, Theorem 1'], [11, Theorem 13.2']; see also [1, Theorem 2.2.2] and [8, Theorem II.4.9], where there is even more general version of this theorem, the so called Calderón-Mitjagin interpolation theorem), we get $\left\|C_{\tau} x\right\|_{\varphi} \leq$ $\max \{K, 1\}\|x\|_{\varphi}$ for all $x \in L^{\varphi}(\Omega)$. More precisely,

$$
\left\|C_{\tau}\right\|_{L^{\varphi} \rightarrow L^{\varphi}} \leq \sup _{u>0} \frac{\varphi^{-1}(K u)}{\varphi^{-1}(u)}
$$

(see [12, Theorem 15.13] or [8, estimate (4.24) on page 100]).

REMARK 3. From the above proofs we obtain that if condition (2.2) holds, then we have the estimates

$$
\begin{aligned}
& \sup _{A \in \Sigma, 0<\mu(A)<\infty} \frac{\varphi^{-1}(1 / \mu(A))}{\varphi^{-1}\left(1 / \mu\left(\tau^{-1}(A)\right)\right)} \\
& \quad \leq\left\|C_{\tau}\right\|_{L^{\varphi} \rightarrow L^{\varphi}} \leq \sup _{u>0} \frac{\varphi^{-1}\left(\sup _{A \in \Sigma, 0<\mu(A)<\infty}\left(\mu\left(\tau^{-1}(A)\right) / \mu(A)\right) u\right)}{\varphi^{-1}(u)},
\end{aligned}
$$

which is a generalization of the $L^{p}$-case into Orlicz spaces.

REMARK 4. Condition (2.2) is sufficient for the continuity of any composition operator from any symmetric space $X$ into itself if $X$ has either the Fatou property or an absolutely continuous norm (see, for example, [1, Theorem 2.2.2] or [8, Theorem II.4.9 and 4.10]), because $X$ is then an interpolation space between $L^{1}$ and $L^{\infty}$.

REMARK 5. If $0<a_{\varphi} \leq b_{\varphi}<\infty$, then the Orlicz space $L^{\varphi}(\Omega)$ is equal to $L^{\infty}(\Omega)$ with an equivalent norm. Hence the composition operator $C_{\tau}$ is norm-continuous on $L^{\varphi}(\Omega)$ for every nonsingular transformation $\tau$. However, in order to obtain modular continuity of $C_{\tau}$ which is stronger than norm continuity, we need the additional assumption (2.2) on $\tau$ as shown in Theorem 2.1. In particular, note that $a_{\varphi}=b_{\varphi}$ is allowed (see [17, first paragraph on page 52]).

THEOREM 2.3. Assume that $\mu$ is a non-atomic infinite measure, $\tau: \Omega \rightarrow \Omega$ is a measurable nonsingular transformation and $\varphi$ is an Orlicz function satisfying the condition $\Delta_{2}$ for large $u$. Then the composition operator $C_{\tau}$ is bounded in $L^{\varphi}(\Omega)$ if and only if there exists a constant $K \geq 1$ such that

$$
\frac{1}{\varphi^{-1}\left(1 / \mu\left(\tau^{-1}(A)\right)\right)} \leq \frac{K}{\varphi^{-1}(1 / \mu(A))}
$$

for all $A \in \Sigma$ with $0<\mu(A)<\infty$. 
PROOF. The necessity is clear by putting $x=\chi_{A}$ into the assumption.

Sufficiency. Since $\varphi$ satisfies the condition $\Delta_{2}$ for large $u$ it follows that $\varphi$ is finite-valued and so $\lim _{u \rightarrow \infty} \varphi^{-1}(u)=\infty$.

Note first that (2.5) guarantee that $\mu \circ \tau^{-1} \ll \mu$. In fact, assume that $\mu(A) \rightarrow 0$. Then by the assumption (2.5) and from the assumption on $\varphi$ we obtain $\mu \circ \tau^{-1}(A) \rightarrow 0$, which shows that $\mu \circ \tau^{-1} \ll \mu$ because by the nonsingularity of $\tau$ we may assume that $\mu(A)>0$. Let $g=d \mu \circ \tau^{-1} / d \mu$. Then $g \in L^{1}+L^{\infty}$ and $\mu \circ \tau^{-1}(A)=\int_{A} g(t) d \mu$ for any $A \in \Sigma$.

Now we will prove that $g \in L^{\infty}(\Omega)$. We can prove in the same way as in the proof of Theorem 2.2 with $2 K$ instead of $K$ that $\varphi \in \Delta_{2}^{\infty}$ (that is, $\varphi$ satisfies the condition $\Delta_{2}$ for large $u$ ) yields $2 K \varphi^{-1}(v) \leq \varphi^{-1}(L v)$ for $v>0$ large enough and an absolute constant $L \geq 2 K$ or equivalently that $2 K \varphi^{-1}(w / L) \leq \varphi^{-1}(w)$ for $w \geq a$ with some $a>0$. We will show that $\|g\|_{\infty} \leq L$. Assume that $\|g\|_{\infty}>L$. Then, as $\mu$ is nonatomic there exists a set $A \in \Sigma$ with $1 / \mu(A)>a$ such that $0<\mu(A)<1 / a$ and $g(t)>L$ for $\mu$-almost all $t \in A$. Consequently,

$$
\mu \circ \tau^{-1}(A)=\int_{A} g(t) d \mu>L \mu(A),
$$

whence $\varphi^{-1}\left(1 / \mu\left(\tau^{-1}(A)\right)\right) \leq \varphi^{-1}(1 / L \mu(A))$ and so

$$
\frac{1}{\varphi^{-1}\left(1 / \mu\left(\tau^{-1}(A)\right)\right)} \geq \frac{1}{\varphi^{-1}(1 / L \mu(A))} \geq \frac{2 K}{\varphi^{-1}(1 / \mu(A))}>\frac{K}{\varphi^{-1}(1 / \mu(A))},
$$

a contradiction. Thus, $\|g\|_{\infty} \leq L$ and the rest of the proof is the same as the proof of the sufficiency in Theorem 2.2 .

REMARK 6. Condition (2.2) implies condition (2.5). Morover, if $\mu(\Omega)<\infty$ and $\varphi$ satisfies the $\Delta_{2}$-condition for large $\mathrm{u}$, then conditions (2.5) and (2.2) are equivalent. In fact, the $\Delta_{2}$-condition of $\varphi$ on $\left[u_{0}, \infty\right)$ can be extended to the interval $\left[\varphi^{-1}\left(1 / \mu\left(\tau^{-1}(\Omega)\right)\right), \infty\right)$ and $(2.5)$ is equivalent to

$$
1 / \mu(A) \leq \varphi\left(K \varphi^{-1}\left(1 / \mu\left(\tau^{-1}(A)\right)\right)\right),
$$

therefore we get condition (2.2). Note that conditions (2.5) and (2.2), in the case when $\mu(\Omega)=\infty$, are different which will be shown in the next theorem.

In our next theorem, the space $L^{\varphi}(\Omega) \cap L^{\infty}(\Omega)$ is considered with the classical norm $\|x\|_{\varphi \cap \infty}=\max \left(\|x\|_{\varphi},\|x\|_{\infty}\right)$.

THEOREM 2.4. Assume that $(\Omega, \Sigma, \mu)$ is a non-atomic infinite measure space and $\tau: \Omega \rightarrow \Omega$ is a measurable nonsingular transformation. Let $\varphi$ be an Orlicz function 
satisfying the condition $\Delta_{2}$ for all $u>0$. Then the composition operator $C_{\tau}$ is bounded in $L^{\varphi}(\Omega) \cap L^{\infty}(\Omega)$ if and only if there exists a constant $K \geq 1$ such that

$$
\max \left\{1, \mu\left(\tau^{-1}(A)\right)\right\} \leq K \max \{1, \mu(A)\}
$$

for all $A \in \Sigma$ with $0<\mu(A)<\infty$.

Proof. Necessity. Putting $x=\chi_{A}$, where $A \in \Sigma$ and $0<\mu(A)<\infty$, we obtain

$$
\begin{aligned}
\max \left\{1, \frac{1}{\varphi^{-1}\left(1 / \mu\left(\tau^{-1}(A)\right)\right)}\right\} & =\max \left\{\left\|C_{\tau} x\right\|_{\infty},\left\|C_{\tau} x\right\|_{\varphi}\right\} \\
& \leq C \max \left\{\|x\|_{\infty},\|x\|_{\varphi}\right\} \\
& =C \max \left\{1, \frac{1}{\varphi^{-1}(1 / \mu(A))}\right\},
\end{aligned}
$$

with a constant $C>0$ independent of $A$. The function $g(u)=1 / \varphi(1 / u)$ is increasing and continuous on $(0, \infty)$ with $\lim _{u \rightarrow 0^{+}} g(u)=0$ and $g(u) \rightarrow \infty$ as $u \rightarrow \infty$. In particular, there exists $a>0$ such that $g(a)=1$. Moreover, $g$ satisfies the condition $\Delta_{2}$ for all $u$ since $\varphi$ is such. Thus

$$
\begin{aligned}
& \max \left\{1, \mu\left(\tau^{-1}(A)\right)\right\} \\
& \quad=\max \left\{g(a), g\left(\frac{1}{\varphi^{-1}\left(1 / \mu\left(\tau^{-1}(A)\right)\right)}\right)\right\}=g\left(\max \left\{a, \frac{1}{\varphi^{-1}\left(1 / \mu\left(\tau^{-1}(A)\right)\right)}\right\}\right) \\
& \leq g\left(C \max \left\{a, \frac{1}{\varphi^{-1}(1 / \mu(A))}\right\}\right) \leq D g\left(\max \left\{a, \frac{1}{\varphi^{-1}(1 / \mu(A))}\right\}\right) \\
& =D \max \left\{g(a), g\left(\frac{1}{\varphi^{-1}(1 / \mu(A))}\right)\right\}=D \max \{1, \mu(A)\},
\end{aligned}
$$

and (2.6) holds.

Sufficiency. We first show that (2.6) implies that $C_{\tau}$ is bounded in $L^{1}(\Omega) \cap L^{\infty}(\Omega)$ and next using an appropriate interpolation theorem we will extend the boundedness of $C_{\tau}$ into $L^{\varphi}(\Omega) \cap L^{\infty}(\Omega)$.

Let $x$ be a simple positive function, that is, $x=\sum_{i=1}^{n} a_{i} \chi_{A_{i}}$, where $A_{1} \subset \cdots \subset A_{n}$ and $a_{i}>0$, and $A_{i} \in \Sigma, \mu\left(A_{i}\right)<\infty, i=1,2, \ldots, n$. Then $C_{\tau} x=\sum_{i=1}^{n} a_{i} \chi_{\tau^{-1}\left(A_{i}\right)}$,

$$
\begin{aligned}
\|x\|_{1} & =\int_{\Omega} \sum_{i=1}^{n} a_{i} \chi_{A_{i}}(t) d \mu(t) \\
& =\sum_{i=1}^{n} a_{i} \mu\left(A_{1}\right)+\sum_{i=2}^{n} a_{i} \mu\left(A_{2} \backslash A_{1}\right)+\cdots+a_{n} \mu\left(A_{n} \backslash A_{n-1}\right)=\sum_{i=1}^{n} a_{i} \mu\left(A_{i}\right),
\end{aligned}
$$


and applying estimate (2.6), we get

$$
\begin{aligned}
\left\|C_{\tau} x\right\|_{1 \cap \infty} & =\max \left(\left\|C_{\tau} x\right\|_{1},\left\|C_{\tau} x\right\|_{\infty}\right) \\
& =\max \left(\left\|\sum_{i=1}^{n} a_{i} \chi_{\tau^{-1}\left(A_{i}\right)}\right\|_{1},\left\|\sum_{i=1}^{n} a_{i} \chi_{\tau^{-1}\left(A_{i}\right)}\right\|_{\infty}\right) \\
& \leq \max \left(\sum_{i=1}^{n} a_{i}\left\|\chi_{\tau^{-1}\left(A_{i}\right)}\right\|_{1}, \sum_{i=1}^{n} a_{i}\right) \\
& \leq \sum_{i=1}^{n} a_{i} \max \left(\mu\left(\tau^{-1}\left(A_{i}\right)\right), 1\right) \leq K \sum_{i=1}^{n} a_{i} \max \left(\mu\left(A_{i}\right), 1\right) \\
& \leq K \sum_{i=1}^{n} a_{i}\left(\mu\left(A_{i}\right)+1\right)=K\left(\|x\|_{1}+\|x\|_{\infty}\right) \\
& \leq 2 K \max \left(\|x\|_{1},\|x\|_{\infty}\right)=2 K\|x\|_{1 \cap \infty} .
\end{aligned}
$$

If $x$ is a simple function in $L^{1} \cap L^{\infty}$, then

$$
\begin{aligned}
\left\|C_{\tau} x\right\|_{1 \cap \infty} & =\left\|C_{\tau} x_{+}-C_{\tau} x_{-}\right\|_{1 \cap \infty} \leq\left\|C_{\tau} x_{+}\right\|_{1 \cap \infty}+\left\|C_{\tau} x_{-}\right\|_{1 \cap \infty} \\
& \leq 2 K\left(\left\|x_{+}\right\|_{1 \cap \infty}+\left\|x_{-}\right\|_{1 \cap \infty}\right) \leq 4 K\|x\|_{1 \cap \infty} .
\end{aligned}
$$

Finally, since simple functions are dense in $L^{1}(\Omega) \bigcap L^{\infty}(\Omega)$ (see [8, page 77]), we have $\left\|C_{\tau} x\right\|_{1 \cap \infty} \leq 4 K\|x\|_{1 \cap \infty}$ for all $x \in L^{1}(\Omega) \bigcap L^{\infty}(\Omega)$ and we proved the boundedeness of the composition operator $C_{\tau}$ in $L^{1}(\Omega) \bigcap L^{\infty}(\Omega)$.

The composition operator $C_{\tau}$ is obviously bounded in $L^{\infty}(\Omega)$ and we showed above that it is also bounded in $L^{1}(\Omega) \bigcap L^{\infty}(\Omega)$ when (2.6) holds. Now, we will show using the interpolation theory (or precisely, the Calderón-Lozanovskii interpolation construction) that $C_{\tau}$ is bounded in $L^{\varphi}(\Omega) \cap L^{\infty}(\Omega)$.

Since $\rho\left(L^{\infty}, L^{1} \cap L^{\infty}\right)=L^{\psi}$, where $\psi^{-1}(t)=\rho(1, \min (1, t)$ ) (see [11, Example 3 or Example 4 and Example 5 on pages 179-181]; see also [14, pages 459-461]) it follows that for $\rho(1, t)=\varphi^{-1}(t)$ we obtain $\psi(u)=\inf \left\{t>0: \psi^{-1}(t)>u\right\}=$ $\inf \left\{t>0: \min \left\{\varphi^{-1}(1), \varphi^{-1}(t)\right\}>u\right\}$, and so

$$
\psi(u)= \begin{cases}\varphi(u) & \text { if } 0 \leq u<\varphi^{-1}(1) \\ \infty & \text { if } u \geq \varphi^{-1}(1) .\end{cases}
$$

Thus $L^{\psi}=L^{\varphi} \cap L^{\infty}$ and the interpolation theorem for Orlicz spaces or the CalderónLozanovskiĭ construction (see [11, Theorem 14.12 or Theorem 15.14]; see also [14, Theorem 8.2.2]) gives that $C_{r}$ is bounded in $\rho\left(L^{\infty}, L^{1} \cap L^{\infty}\right)=L^{\varphi} \cap L^{\infty}$.

REMARK 7. Under the same assumptions on the measure $\mu$ and the Orlicz function $\varphi$ as in Theorem 2.4 we can prove, similarly as in Theorem 2.4 , that the composition 
operator $C_{\mathrm{r}}$ is bounded in $L^{\varphi}(\Omega)+L^{\infty}(\Omega)$ if and only if there exists a constant $K \geq 1$ such that $\min \left\{1, \mu\left(\tau^{-1}(A)\right)\right\} \leq K \min \{1, \mu(A)\}$ for all $A \in \Sigma$ with $0<\mu(A)<\infty$.

We formulate a necessary and sufficient condition for boundedness of composition operators in Orlicz spaces $L^{\varphi}(\Omega)$ in terms of the embedding of these Orlicz spaces into weighted Orlicz spaces $L_{w}^{\varphi}(\Omega)$ with the norm

$$
\|x\|_{\varphi, w}=\inf \left\{\lambda>0: \int_{\Omega} \varphi\left(\frac{|x(t)|}{\lambda}\right) w(t) d \mu \leq 1\right\},
$$

where the weight $w$ is the Radon-Nikodým derivative of $\mu \circ \tau^{-1}$ with respect to $\mu$.

THEOREM 2.5. Let $(\Omega, \Sigma, \mu)$ be a $\sigma$-finite nonatomic measure space and $\tau: \Omega \rightarrow \Omega$ be a measurable nonsingular transformation with $\tau(\Omega)=\Omega$. Denote by $w$ the RadonNikodým derivative $d \mu \circ \tau^{-1} / d \mu$. Then the following conditions are equivalent:

(a) The composition operator $C_{\tau}$ is bounded from $L^{\varphi}(\Omega)$ into itself.

(b) For every $x \in L^{\varphi}(\Omega)$, there exists $\lambda>0$ such that

$$
\int_{\Omega} \varphi(\lambda|x(t)|) w(t) d \mu(t)<\infty .
$$

(c) The Orlicz space $L^{\varphi}(\Omega)$ is embedded continuously into the weighted Orlicz space $L_{w}^{\varphi}(\Omega)$.

(d) There are $a, b>0$ and $0 \leq g \in L^{1}(\Omega)$ such that $\varphi(a u) w(t) \leq b \varphi(u)+g(t)$ for all $u>0$ and $t \in \Omega \backslash A$ with $\mu(A)=0$.

PROOF. Since $\tau(\Omega)=\Omega$ it follows that

$$
I_{\varphi}\left(C_{\tau} x\right)=I_{\Phi}(x),
$$

where $\Phi(t, u)=\varphi(u) w(t)$ is the Musielak-Orlicz function (see, for example, [13]). In fact,

$$
\begin{aligned}
I_{\varphi}\left(C_{\tau} x\right) & =\int_{\Omega} \varphi\left(\left|C_{\tau} x(t)\right|\right) d \mu(t)=\int_{\Omega} \varphi(|x(\tau(s))|) d \mu(s) \\
& =\int_{\tau(\Omega)} \varphi(|x(t)|) d\left(\mu \circ \tau^{-1}(t)\right)=\int_{\tau(\Omega)} \varphi(|x(t)|) w(t) d(\mu(t)) \\
& =\int_{\Omega} \varphi(|x(t)|) w(t) d \mu(t)=I_{\Phi}(x) .
\end{aligned}
$$

From (2.7) it follows that $C_{\tau}$ is an isometry from $L^{\Phi}(\Omega)$ into $L^{\varphi}(\Omega)$. Namely, defining

$$
\|x\|_{\Phi}=\inf \left\{\lambda>0: \int_{\Omega} \Phi\left(t, \frac{|x(t)|}{\lambda}\right) d \mu \leq 1\right\},
$$


we have for any $x \in L^{\Phi}(\Omega) \backslash\{0\}$, that $I_{\varphi}\left(C_{\tau} x /\|x\|_{\Phi}\right)=I_{\Phi}\left(x /\|x\|_{\Phi}\right) \leq 1$, whence $\left\|C_{\tau} x /\right\| x\left\|_{\Phi}\right\|_{\varphi} \leq 1$, or, equivalently, $\left\|C_{\tau} x\right\|_{\varphi} \leq\|x\|_{\Phi}$. On the other hand, if $C_{\tau} x \in$ $L^{\varphi} \backslash\{0\}$, then $I_{\Phi}\left(x /\left\|C_{\tau} x\right\|_{\varphi}\right)=I_{\varphi}\left(C_{\tau} x /\left\|C_{\tau} x\right\|_{\varphi}\right) \leq 1$, whence $\|x\|_{\Phi} \leq\left\|C_{\tau} x\right\|_{\varphi}$, and the proof that $C_{\tau}$ is an isometry from $L^{\Phi}(\Omega)$ into $L^{\varphi}(\Omega)$ is finished.

The continuity of the operator $C_{\tau}$ in $L^{\varphi}(\Omega)$ means that there exists a constant $K \geq 1$ such that $\|x\|_{\Phi} \leq K\|x\|_{\varphi}$ for all $x \in L^{\varphi}(\Omega)$. This gives a continuous embedding of $L^{\varphi}(\Omega)$ into $L^{\Phi}(\Omega)$.

It is well known that the last embedding holds if and only if condition (d) is satisfied (see [13, Theorem 8.5] for the finite valued functions $\varphi, \Phi$ and $[6,3,18]$ in the arbitrary case). Consequently, we get implications (a) $\Rightarrow(b) \Rightarrow$ (c) $\Rightarrow$ (d). Moreover, condition (d) yields easily condition (a), so the proof is finished.

If the measure space $(\Omega, \Sigma, \mu)$ is $\sigma$-finite and purely atomic, that is, $\Omega=\bigcup_{n=1}^{\infty} A_{n}$, where $A_{n}$ are the atoms with the measures $\mu\left(A_{n}\right)=a_{n}>0$ for all $n \in \mathbb{N}$, then the Orlicz sequence space $l^{\varphi}\left(\left\{a_{n}\right\}\right)$ is defined as the space of all real sequences $x=\left\{x_{n}\right\}_{n=1}^{\infty}$ such that $I_{\varphi}\left(\lambda x,\left\{a_{n}\right\}\right)<\infty$ for some $\lambda>0$, where $I_{\varphi}\left(x,\left\{a_{n}\right\}\right)=\sum_{n=1}^{\infty} \varphi\left(\left|x_{n}\right|\right) a_{n}$. This space is considered with the norm

$$
\|x\|_{\varphi,\left\{a_{n}\right\}}=\inf \left\{\lambda>0: l_{\varphi}\left(x / \lambda,\left\{a_{n}\right\}\right) \leq 1\right\} .
$$

THEOREM 2.6. Let $(\Omega, \Sigma, \mu)$ be a $\sigma$-finite and purely atomic measure space with atoms $\left\{A_{n}\right\}$ of measure $\mu\left(A_{n}\right)=a_{n}>0$ for any $n \in \mathbb{N}$. Let $\tau: \Omega \rightarrow \Omega$ be a nonsingular transformation with $\tau(\Omega)=\Omega$ and $b_{n}:=\mu\left(\tau^{-1}\left(A_{n}\right)\right) / \mu\left(A_{n}\right)$. Then the following conditions are equivalent:

(a) The composition operator $C_{\tau}$ is bounded from $l^{\varphi}\left(\left\{a_{n}\right\}\right)$ into itself.

(b) For every $x \in l^{\varphi}\left(\left\{a_{n}\right\}\right)$ there exists $\lambda>0$ such that $\sum_{n=1}^{\infty} \varphi\left(\lambda\left|x_{n}\right|\right) b_{n}<\infty$.

(c) The Orlicz space $l^{\varphi}\left(\left\{a_{n}\right\}\right)$ is embedded continuously into the Orlicz space $l^{\varphi}\left(\left\{a_{n} b_{n}\right\}\right)$.

(d) There are $a, b, \delta>0$ and a sequence $\left\{c_{n}\right\}$ in $l^{1}$ of nonnegative numbers such that $\varphi(u) a_{n}<\delta \Rightarrow \varphi(a u) a_{n} b_{n} \leq b \varphi(u) a_{n}+c_{n}$ for all $n \in \mathbb{N}$ and all $u>0$.

PROOF. It is very similar to the proof of Theorem 2.5, but we present it for the sake of completeness. We have

$$
\begin{aligned}
I_{\varphi}\left(C_{\tau} x,\left\{a_{n}\right\}\right) & =\sum_{n=1}^{\infty} \varphi\left(\left|C_{\tau} x_{n}\right|\right) a_{n}=\sum_{n=1}^{\infty} \varphi\left(\left|x_{\tau(n)}\right|\right) a_{n} \\
& =\sum_{n \in \tau(\Omega)} \varphi\left(\left|x_{n}\right|\right) \mu\left(\tau^{-1}\left(A_{n}\right)\right)=\sum_{n=1}^{\infty} \varphi\left(\left|x_{n}\right|\right) a_{n} b_{n}=I_{\varphi}\left(x,\left\{a_{n} b_{n}\right\}\right) .
\end{aligned}
$$

For any $x \in l^{\varphi}\left(\left\{a_{n} b_{n}\right\}\right) \backslash\{0\}$,

$$
I_{\varphi}\left(\frac{C_{r} x}{\|x\|_{\varphi,\left\{a_{n} b_{n}\right\}}},\left\{a_{n}\right\}\right)=I_{\varphi}\left(\frac{x}{\|x\|_{\varphi,\left\{a_{n} b_{n}\right\}}},\left\{a_{n} b_{n}\right\}\right) \leq 1
$$


whence $\left\|C_{\tau} x /\right\| x\left\|_{\varphi,\left(a_{n} b_{n}\right\}}\right\|_{\varphi,\left\{a_{n}\right\}} \leq 1$ or, equivalently, $\left\|C_{\tau} x\right\|_{\varphi,\left\{a_{n}\right\}} \leq\|x\|_{\varphi,\left\{a_{n} b_{n}\right\}}$. On the other hand, if $C_{\tau} x \in l^{\varphi}\left(\left\{a_{n}\right\}\right) \backslash\{0\}$, then

$$
I_{\varphi}\left(\frac{x}{\left\|C_{\tau} x\right\|_{\varphi,\left\{a_{n}\right\}}},\left\{a_{n} b_{n}\right\}\right)=I_{\varphi}\left(\frac{C_{\tau} x}{\left\|C_{\tau} x\right\|_{\varphi,\left\{a_{n}\right.}},\left\{a_{n}\right\}\right) \leq 1,
$$

whence $\|x\|_{\varphi,\left\{a_{n} b_{n}\right\}} \leq\left\|C_{\tau} x\right\|_{\varphi,\left\{a_{n}\right\}}$. Consequently, the equality $\left\|C_{\tau} x\right\|_{\varphi,\left\{a_{n}\right\}}=\|x\|_{\varphi,\left\{a_{n} b_{n}\right\}}$ is proved and it shows that $C_{\tau}$ is an isometry from $l^{\varphi}\left(\left\{a_{n} b_{n}\right\}\right)$ into $l^{\varphi}\left(\left\{a_{n}\right\}\right)$. Taking into account that conditions (c) and (d) are equivalent (see [13, Theorem 8.11, page 51] for the finite valued functions and [19] in the arbitrary case), we can finish the proof in the same way as the proof of Theorem 2.5.

\section{Compactness of composition operators in Orlicz spaces}

We start with the following result.

THEOREM 3.1. Let $\varphi$ be an Orlicz function vanishing only at zero with finite values, that is, $a_{\varphi}=0$ and $b_{\varphi}=\infty$. Let $\tau$ be a measurable nonsingular transformation from $\Omega$ into itself such that $\tau(\Omega)=\Omega$. If $C_{\tau}$ is a compact operator from $L^{\varphi}$ into itself, then the measure $\mu$ is purely atomic.

Proof. We can write $\Omega=\Omega_{1} \cup \Omega_{2}$, where $\Omega_{1} \cap \Omega_{2}=\emptyset,\left.\mu\right|_{\Omega_{1}}$ is nonatomic and $\left.\mu\right|_{\Omega_{2}}$ is purely atomic. Since $\mu \circ \tau^{-1} \ll \mu$, then by the Radon-Nikodým theorem there exists a function h locally integrable on $\Omega_{1}$ such that $\mu \circ \tau^{-1}(A)=\int_{A} h(t) d \mu$ for any $A \in \Sigma \cap \Omega_{1}$. Define $A_{0}=\left\{t \in \Omega_{1}: h(t)>0\right\}$. We will show that $\mu \circ \tau^{-1}\left(A_{0}\right)=0$. Assume for the contrary that $\mu \circ \tau^{-1}\left(A_{0}\right)>0$. Then there is $\epsilon>0$ such that the set $A_{1}=\left\{t \in A_{0}: h(t) \geq \epsilon\right\}$ has positive measure. Take a sequence $\left\{B_{n}\right\}$ of pairwise disjoint subsets of $\Sigma \cap A_{1}$ with $0<\mu\left(B_{n}\right)<1 / 2^{n}$ for $n \in \mathbb{N}$ large enough $\left(n>n_{0}\right)$. Define

$$
x_{n}=\varphi^{-1}\left(\frac{1}{\mu\left(B_{n}\right)}\right) \chi_{B_{n}}, \quad n>n_{0} .
$$

Then $I_{\varphi}\left(x_{n}\right)=1$, whence $x_{n} \in L^{\varphi}(\Omega)$ and $\left\|x_{n}\right\|_{\varphi}=1$ for $n>n_{0}$. Consequently, we have for $m, n>n_{0}$ with $m \neq n$,

$$
\begin{aligned}
I_{\varphi}\left(C_{\tau} x_{m}-C_{\tau} x_{n}\right) & =\int_{\Omega} \varphi\left(\left|C_{\tau} x_{m}(s)-C_{\tau} x_{n}(s)\right|\right) d \mu(s) \\
& =\int_{\Omega} \varphi\left(\left|x_{m}(\tau(s))-x_{n}(\tau(s))\right|\right) d \mu(s) \\
& =\int_{\tau(\Omega)} \varphi\left(\left|x_{m}(t)-x_{n}(t)\right|\right) d \mu \circ \tau^{-1}(t)
\end{aligned}
$$




$$
\begin{aligned}
& =\int_{\Omega} \varphi\left(\left|x_{m}(t)-x_{n}(t)\right|\right) d \mu \circ \tau^{-1}(t) \\
& =\int_{\Omega} \varphi\left(\left|x_{m}(t)-x_{n}(t)\right|\right) h(t) d \mu(t) \\
& =\int_{B_{m}} \varphi\left(\left|x_{m}(t)\right|\right) h(t) d \mu(t)+\int_{B_{n}} \varphi\left(\left|x_{n}(t)\right|\right) h(t) d \mu(t) \\
& \geq \frac{1}{\mu\left(B_{m}\right)} \epsilon \mu\left(B_{m}\right)+\frac{1}{\mu\left(B_{n}\right)} \epsilon \mu\left(B_{n}\right)=2 \epsilon .
\end{aligned}
$$

Therefore, $\left\|C_{\tau} x_{m}-C_{\mathrm{r}} x_{n}\right\|_{\varphi} \geq 2 \epsilon$ for $m, n>n_{0}$ with $m \neq n$. This means that $\left\{C_{\tau} x_{n}\right\}$ contains no subsequence which is a Cauchy sequence, that is, $C_{\tau}\left(B\left(L^{\varphi}(\Omega)\right)\right)$, where $B\left(L^{\varphi}(\Omega)\right)$ denotes the unit ball of $L^{\varphi}(\Omega)$, is not relatively compact. Consequently, the operator $C_{\tau}$ is not compact, a contradiction. The assumption that the transformation $\tau$ is nonsingular yields that $\mu\left(A_{0}\right)=0$. The proof of the theorem is finished.

THEOREM 3.2. Let $\varphi$ be a finite-valued Orlicz function and let $(\Omega, \Sigma, \mu)$ be a $\sigma$-finite and purely atomic measure space with the atoms $A_{n}$ of measure $\mu\left(A_{n}\right)=$ $a_{n}>0$. For a measurable nonsingular transformation $\tau$ from $\Omega$ into itself, denote $b_{n}:=\mu\left(\tau^{-1}\left(A_{n}\right)\right) / \mu\left(A_{n}\right)$. If $C_{\tau}$ is a compact operator from $l^{\varphi}\left(\left\{a_{n}\right\}\right)$ into itself, then $\lim _{n \rightarrow \infty} b_{n}=0$.

ProOF. Assume for the contrary that the assumptions are satisfied and $b_{n} \nrightarrow 0$. We may assume without loss of generality that there is $\epsilon \epsilon(0,1)$ such that $b_{n} \geq \epsilon$ for all $n \in \mathbb{N}$. Define

$$
x_{n}=\varphi^{-1}\left(\frac{1}{\mu\left(\tau^{-1}\left(A_{n}\right)\right)}\right) \chi_{A_{n}}, \quad n \in \mathbb{N} \text {. }
$$

Then

$$
C_{\tau} x_{n}=\varphi^{-1}\left(\frac{1}{\mu\left(\tau^{-1}\left(A_{n}\right)\right)}\right) \chi_{\tau^{-1}\left(A_{n}\right)}
$$

and

$$
I_{\varphi}\left(x_{n}\right) \leq \frac{\mu\left(A_{n}\right)}{\mu\left(\tau^{-1}\left(A_{n}\right)\right)}=\frac{1}{b_{n}} \leq \frac{1}{\epsilon}<\infty,
$$

whence $\left\|x_{n}\right\|_{\varphi} \leq 1 / \epsilon$ for all $n \in \mathbb{N}$.

On the other hand, we have for all $m \neq n$,

$$
\begin{aligned}
I_{\varphi}\left(C_{\tau} x_{m}-C_{\tau} x_{n}\right) & =I_{\varphi}\left(C_{\tau}\left(x_{m}-x_{n}\right)\right) \\
& =\frac{1}{\mu\left(\tau^{-1}\left(A_{m}\right)\right)} \mu\left(\tau^{-1}\left(A_{m}\right)\right)+\frac{1}{\mu\left(\tau^{-1}\left(A_{n}\right)\right)} \mu\left(\tau^{-1}\left(A_{n}\right)\right)=2 .
\end{aligned}
$$

Consequently, $\left\|C_{\tau} x_{m}-C_{\tau} x_{n}\right\|_{\varphi}>1$ for all $m \neq n$, which means that $\left\{C_{\tau} x_{n}\right\}$ contains no Cauchy subsequence, that is, $C_{\tau}$ is not compact. This contradiction finishes the proof. 
THEOREM 3.3. Let $\varphi$ be an Orlicz function vanishing only at zero. Let $(\Omega, \Sigma, \mu)$ be a purely atomic $\sigma$-finite measure space withe the atoms $A_{n}$ of measure $\mu\left(A_{n}\right)=$ $x_{n}>0$ and $\tau$ be a measurable nonsingular transformation from $\Omega$ into itself such that $\lim _{n \rightarrow \infty} b_{n}=0$. Assume that either $\varphi$ satisfies condition $\Delta_{2}$ for all $u>0$ when $\sum_{n=1}^{\infty} b_{n}=\infty$ or condition $\Delta_{2}$ for large $u>0$ when $\sum_{n=1}^{\infty} b_{n}<\infty$. Then the composition operator $C_{\tau}$ acts from $l^{\varphi}\left(\left\{a_{n}\right\}\right)$ into itself and it is compact.

PROOF. The assumption on $\varphi$ implies that (see [2, Theorem 1.39], [13])

(3.1) for any $\epsilon>0$ there exists $\delta>0$ such that $I_{\varphi}(x)<\delta$ implies $\|x\|_{\varphi}<\epsilon$.

Let $\left\{x_{n}\right\}$ be a sequence from $B\left(l^{\varphi}\left(\left\{a_{n}\right\}\right)\right)$. Then there exists a subsequence $\left\{x_{n_{k}}\right\}$ of $\left\{x_{n}\right\}$ and $x \in l^{\varphi}\left(\left\{a_{n}\right\}\right)$ such that $\left\{x_{n_{k}}\right\}$ is weakly* convergent to $x$. We may assume without loss of generality that $x_{n} \rightarrow 0$ weakly $^{*}$ (if $x \neq 0$ we consider the sequence $\left\{x_{n}-x\right\}$ instead of $\left\{x_{n}\right\}$ ). Since $b_{i} \rightarrow 0$ as $i \rightarrow \infty$, there is $j \in \mathbb{N}$ such that $b_{i}<\delta / 2$ for all $i>j$. Then

$$
\sum_{i=j+1}^{\infty} b_{i} \varphi\left(\left|x_{n}(i)\right|\right) \mu\left(A_{i}\right)<\delta / 2 \sum_{i=j+1}^{\infty} \varphi\left(\left|x_{n}(i)\right|\right) \mu\left(A_{i}\right) \leq \delta / 2
$$

for all $n \in \mathbb{N}$. Note that if $x_{n} \rightarrow 0$ weakly*, then $x_{n} \rightarrow 0$ pointwise. Really, the predual of $l^{\varphi}\left(\left\{a_{n}\right\}\right)$ is the subspace $h^{\psi}$ of order continuous elements of the MusielakOrlicz sequence space $l^{\Psi}$ over the counting measure with the Musielak-Orlicz function $\Psi=\left\{\psi_{n}\right\}_{n=1}^{\infty}$, where $\psi_{n}(u)=a_{n} \varphi^{*}\left(u / a_{n}\right)$ for all $u>0$ and $n \in \mathbb{N}$. Take any $k \in \mathbb{N}$. Then $y=e_{k} \in h^{\Psi}$. We have $x_{n}(k)=\left\langle x_{n}, y\right\rangle \rightarrow 0$ by assumption, which means that $x_{n} \rightarrow 0$ pointwise.

Thus, there exists $m \in \mathbb{N}$ such that $\sum_{i=1}^{j} b_{i} \varphi\left(\left|x_{n}(i)\right|\right) \mu\left(A_{i}\right)<\delta / 2$ for all $n>m$. Combining the above two estimates we get

$$
I_{\varphi}\left(C_{\tau} x_{n}\right)=\sum_{i=1}^{\infty} \varphi\left(\left|x_{n}(i)\right|\right) \mu \tau^{-1}\left(A_{i}\right)=\sum_{i=1}^{\infty} \varphi\left(\left|x_{n}(i)\right|\right) b_{i} \mu\left(A_{i}\right)<\delta
$$

for $n>m$. Applying condition (3.1), we obtain $\left\|C_{\tau} x_{n}\right\|_{\varphi}<\epsilon$ for all $n>m$, which means that $C_{\tau} x_{n} \rightarrow 0$. The proof is complete.

The following example shows that in the last theorem the assumptions that $\varphi$ vanishes only at zero and that $\varphi$ satisfies a suitable $\Delta_{2}$-condition are important.

EXAMPLE 1. Let $\varphi(u)=0$ if $0 \leq u \leq 1$ and $\varphi(u)=\infty$ if $u>1$. Then for any measure space $(\Omega, \Sigma, \mu)$, we have $L^{\varphi}(\Omega, \Sigma, \mu)=L^{\infty}(\Omega, \Sigma, \mu)$ with equality of the norms $\|x\|_{\varphi}$ and $\|x\|_{\infty}$. Thus in the case of the counting measure space, we have 
$\|x\|_{\varphi}=\|x\|_{\infty}=\sup _{i \in \mathrm{N}}|x(i)|$. It is easy to see that the only compact composition operators $C_{r}: l^{\infty}(\mathbb{N}) \rightarrow l^{\infty}(\mathbb{N})$ are finitely dimensional operators.

Assume for the contrary that $C_{\tau}$ is not finite dimensional. Then we may assume without loss of generality that $C_{\tau} \chi_{A_{n}} \neq 0$ for any atom $A_{n}$. Thus $\chi_{\tau-1}\left(A_{n}\right) \neq 0$ for any atom $A_{n}$, which means that $\mu\left(\tau^{-1}\left(A_{n}\right)\right) \neq 0$, whence, $\tau^{-1}\left(A_{n}\right) \neq \emptyset$ for all $n \in \mathbb{N}$. Defining $x_{n}=\chi_{A_{n}}$, we have $\left\|x_{n}\right\|_{\infty}=1$ for all $n \in \mathbb{N}$ and if $m \neq n$, we get $\left\|C_{\tau} x_{m}-C_{\tau} x_{m}\right\|_{\infty}=1$, which means that $C_{\tau}$ is not compact.

THEOREM 3.4. Let $\varphi$ be an Orlicz function vanishing only at zero with $b_{\varphi}<\infty$ and $\varphi_{-}^{\prime}\left(b_{\varphi}\right)<\infty$, where $\varphi_{-}^{\prime}$ stands for the left derivative of $\varphi$. Assume that $(\Omega, \Sigma, \mu)$ is a purely atomic $\sigma$-finite measure space with the atoms $A_{n}$ of measure $\mu\left(A_{n}\right)=a_{n}>0$ such that $\liminf _{n \rightarrow \infty} \mu\left(A_{n}\right) \geq \alpha>0$. Assume that $\tau$ is a measurable nonsingular transformation from $\Omega$ into itself. If $C_{\tau}$ is a compact operator from $l^{\varphi}\left(\left\{a_{n}\right\}\right)$ into itself, then $\lim _{n \rightarrow \infty} b_{n}=0$.

PROOF. We may assume without loss of generality that $b_{\varphi}=1$. Otherwise we may consider the function $\varphi_{1}(u)=\varphi\left(b_{\varphi} u\right)$, for which $L^{\varphi_{1}}(\Omega)=L^{\varphi}(\Omega)$ and $\|\cdot\|_{\varphi_{1}}=b_{\varphi}\|\cdot\|_{\varphi}$. There is a finite-valued Orlicz function $\psi$ such that $\psi(u)=\varphi(u)$ for $0 \leq u \leq 1$. Define

$$
\varphi_{\infty}(u)= \begin{cases}0 & \text { if } 0 \leq u \leq 1 \\ \infty & \text { if } u>1\end{cases}
$$

Then $\varphi=\max \left\{\psi, \varphi_{\infty}\right\}$. Therefore (see [11, Theorem 12.2] and [17, page 130]) $L^{\varphi}(\Omega)=L^{\psi}(\Omega) \bigcap L^{\infty}(\Omega)$ and $\|x\|_{\varphi}=\max \left\{\|x\|_{\psi},\|x\|_{\infty}\right\}$ for all $x \in L^{\varphi}(\Omega)$.

Assume for the contrary that $b_{n} \nrightarrow 0$ as $n \rightarrow \infty$. Then we may assume without loss of generality that there is $\epsilon>0$ such that $b_{n} \geq \epsilon$ and $w_{n}=\mu\left(A_{n}\right) \geq \alpha>0$ for all $n \in \mathbb{N}$. Define $x_{n}=\min \left\{1, \psi^{-1}\left(1 / w_{n}\right)\right\} \chi_{A_{n}}, n=1,2, \ldots$ Since

$$
I_{\varphi}\left(x_{n}\right)=I_{\psi}\left(x_{n}\right)=\psi\left(\min \left\{1, \psi^{-1}\left(1 / w_{n}\right)\right\}\right) \mu\left(A_{n}\right) \leq \frac{1}{w_{n}} w_{n}=1,
$$

it follows that $\left\|x_{n}\right\|_{\varphi} \leq 1$ for all $n \in \mathbb{N}$. On the other hand, we have for $m \neq n$,

$$
\begin{aligned}
I_{\varphi}\left(C_{\tau} x_{m}-C_{\tau} x_{n}\right)= & I_{\psi}\left(C_{\tau} x_{m}-C_{\tau} x_{n}\right) \\
= & \psi\left(\min \left\{1, \psi^{-1}\left(1 / w_{m}\right)\right\}\right) \mu\left(\tau^{-1}\left(A_{m}\right)\right) \\
& +\psi\left(\min \left\{1, \psi^{-1}\left(1 / w_{n}\right)\right\}\right) \mu\left(\tau^{-1}\left(A_{n}\right)\right) \\
\geq & \epsilon \psi\left(\min \left\{1, \psi^{-1}\left(1 / w_{m}\right)\right\}\right) w_{m}+\epsilon \psi\left(\min \left\{1, \psi^{-1}\left(1 / w_{n}\right)\right\}\right) w_{n} \\
\geq & \epsilon \min \left\{\psi(1), 1 / w_{m}\right\} w_{m}+\epsilon \min \left\{\psi(1), 1 / w_{n}\right\} w_{n} \\
\geq & 2 \epsilon \min \{\alpha \psi(1), 1\}>0 .
\end{aligned}
$$

This means that the operator $C_{\tau}$ is not compact which is a contradiction. 


\section{Acknowledgements}

The authors are grateful to the anonymous referee for her/his careful reading of the paper and for various valuable suggestions and improvements. Research of the first named author was supported by the NSF of China. Research of the third named author was supported by NBHM travel grant No 44/7/2001-R and D-II/317. Research of the fourth named author was supported by the Swedish Natural Science Research Council (NFR) - grant M5105-20005228/2000 and also by the Swedish Institute (Visby Programme No. 3773/2001).

\section{References}

[1] C. Bennett and R. Sharpley, Interpolation of operators (Academic Press, London, 1988).

[2] S. Chen, Geometry of Orlicz spaces, Dissertationes Math. (Rozprawy Mat.) 356 (PWN, Warsaw, 1996).

[3] L. Drewnowski and W. Orlicz, 'A note on modular spaces. XI', Bull. Acad. Polon. Sci. Sér. Sci. Math. Astronom. Phys. 16 (1968), 877-882.

[4] N. Dunford and J. T. Schwartz, Linear operators, 1. General theory (Interscience, New York, 1958).

[5] H. Hudzik and L. Maligranda, 'Amemiya norm equals Orlicz norm in general', Indag. Math. N. S. $11(2000)$, 573-585.

[6] J. Ishii, 'On equivalence of modular function spaces', Proc. Japan Acad. 35 (1959), 551-556.

[7] M. A. Krasnoselskii and Ya. B. Rutickii, Convex functions and Orlicz spaces (Noordhoff, Groningen, 1961).

[8] S. G. Krein, Ju. I. Petunin and E. M. Semenov, Interpolation of linear operators, Translations of Mathematical Monographs 54 (Amer. Math. Soc., Providence, 1982).

[9] R. Kumar, 'Composition operators on Orlicz spaces', Integral Equations Operator Theory 29 (1997), 17-22.

[10] J. Lindenstrauss and L. Tzafriri, Classical Banach spaces II. Function spaces (Springer, Berlin, 1979).

[11] L. Maligranda, Orlicz spaces and interpolation, Seminars in Math. 5 (Univ. Estadual de Campinas, Campinas SP, Brazil, 1989).

[12] __ ' 'Some remarks on Orlicz's interpolation theorem', Studia Math. 95 (1989), 43-58.

[13] J. Musielak, Orlicz spaces and modular spaces, Lecture Notes in Math. 1034 (Springer, Berlin, 1983).

[14] V. I. Ovchinnikov, 'The methods of orbits in interpolation theory', Math. Rep. 1 (1984), 349-516.

[15] S. Petrović, 'A note on composition operators', Mat. Vesnik 40 (1988), 147-151.

[16] M. M. Rao, 'Convolutions of vector fields-II: random walk models', Nonlinear Anal., Theory Methods Appl. 47 (2001), 3599-3615.

[17] M. M. Rao and Z. D. Ren, Theory of Orlicz spaces (Marcel Dekker, New York, 1991).

[18] I. V. Shragin, 'The operator of superposition in modular function spaces', Studia Math. 43 (1972), 61-75 (Russian).

[19] —_, 'Conditions for the imbedding of classes of sequences, and their consequences', Mat. Zametki 20 (1976), 681-692, English translation: Math. Notes 20 (1976), 942-948 (1977).

[20] R. K. Singh, 'Compact and quasinormal composition operators', Proc. Amer. Math. Soc. 45 (1974), 80-82. 
[21] — 'Composition operators induced by rational functions', Proc. Amer. Math. Soc. 59 (1976), 329-333.

[22] R. K. Singh and R. D. Chandra Kumar, 'Compact weighted composition operators on $L^{2}(\lambda)$ ', Acta Sci. Math. (Szeged) 49 (1985), 339-344.

[23] R. K. Singh and B. S. Komal, 'Composition operator on $\ell^{p}$ and its adjoint', Proc. Amer. Math. Soc. $70(1978), 21-25$.

[24] R. K. Singh and A. Kumar, 'Compact composition operators', J. Austral. Math. Soc. Ser. A 28 (1979), 309-314.

[25] R. K. Singh and J. S. Manhas, Composition operators on function spaces, North-Holland Math. Studies 179 (North-Holland, Amsterdam, 1993).

[26] H. Takagi, 'Compact weighted composition operators on $L^{p}$ ', Proc. Amer. Math. Soc. 116 (1992), 505-511.

[27] H. Takagi and K. Yokouchi, 'Multiplication and composition operators between two $L^{p}$-spaces', in: Function spaces, Edwardsville, IL, 1998, Contemp. Math. 232 (Amer. Math. Soc., Providence, 1999) pp. 321-338.

[28] X. M. Xu, 'Compact composition operators on $L^{p}(X, \mathscr{A}, \mu)$ ', Adv in Math. (China) (2) 20 (1991), 221-225 (Chinese).

Department of Mathematics

Harbin University of Sciences and Technology

52 Xuefu Road, Nanang. Dist.

Harbin, Heilongjiang 150080 P. R. of China

e-mail: cuiya@mail.hrbust.edu.cn
Faculty of Mathematics and Computer Science Adam Mickiewicz University Umultowska 87 61-614 Poznan

Poland e-mail: hudzik@amu.edu.pl
Department of Mathematics

University of Jammu

Jammu-180 004

India

e-mail: romesh_jammu@yahoo.com
Department of Mathematics

Luleå University of Technology

SE-97187 Luleå

Sweden

e-mail: lech@sm.luth.se 